\title{
The influence of geometrical tolerances of Vickers indenter on the accuracy of measured hardness
}

\author{
M. El-Sherbiny ${ }^{1}$, R. Hegazy ${ }^{2, \star, \star \star}$, M. Ibrahim ${ }^{2}$, and A. Abuelezz ${ }^{2}$ \\ 1 Faculty of Engineering, Cairo University, Gamaet El Qahera St. Giza, Giza, Egypt \\ 2 National Institute for Standards (NIS), 136 Tersa St., Mahatet El Matbaa, EL HARAM, Cairo, Egypt
}

Received: 27 March 2012 / Accepted: 27 March 2012

\begin{abstract}
This paper reports work carried out to determine the influence of the Pyramidal indenter geometrical errors and tolerances on the accuracy of Vickers hardness measurements. The angle between the opposite faces and line of conjunction of nine indenters were measured by the optical comparator. The influence of the indenter geometry on the hardness accuracy is investigated. Three calibrated reference hardness test blocks were used to check the Vickers hardness test using the nine selected indenters. The results show that the geometrical error of the indenters (specially the angles) considerably affects the accuracy of the hardness measurement. Finite element analysis of the indentation process into metallic test pieces was conducted. Comparison of the experimental results with the numerical findings was discussed. Errors in the experimental values are correlated with measured tolerances in the angles between faces.
\end{abstract}

Keywords: Vickers indenter; hardness; geometrical errors; tolerances; accuracy

\section{Introduction}

The main reasons for differences in hardness measurement are due to indenter effects. The replacement of an indenter represents a new hardness scale as illustrated by the replacement of Rockwell indenters at National Physical Laboratory (NPL) [1] and the Etablissement Technique Central de l'Armement (ETCA) in France [2]. It has been shown that, provided the same diamond indenter was used, machines of quite different designs were capable of giving the same hardness scales. Whereas, if more than one indenter was involved, limited agreement between machines was observed [3]. This was attributed to the poor traceability in characterizing indenters and, therefore, poor reproducibility when changing indenters. In addition, continuous use of an indenter can cause a drift in the characteristics of an indenter and thus cause a change in the hardness scale [4].

Transparency is essential for all accredited laboratories involved in calibration metrology and certification [5]. This means that procedures, techniques and reference standards used to establish a hardness scale must be documented such that they can be reproduced in other standards laboratories.

An extensive investigation at National Physical Laboratory [6] was carried out to investigate the influence of indenter morphology on the performance of Rockwell HRC, HRA and HR $30 \mathrm{~N}$ scales. The effect of indenter radius was similar for all scales, a reduction in the radius resulted in an apparent softening of the test block, whilst an increase in the radius resulted in an apparent increased

\footnotetext{
* Correspondence: rihamhegazy@yahoo.com

${ }^{\star \star}$ Researcher in National Institute for Standards
}

hardness, and that the indenter radius was more critical when using harder test blocks.

Wood et al. [7] recommend further studies to investigate the influence of the reduced tolerances of indenter radius and cone angle for both calibration and standard Rockwell indenters on variation in hardness measurement and its contribution to hardness uncertainty. Investigations at National Institute of Standards and Technology [8-10] have identified the key characteristics of Rockwell diamond indenters and concluded that both geometric and non-geometric properties affect the hardness performance.

The main advantages of the Vickers hardness test are that accurate readings can be taken, and just one type of indenter is used for all types of metals and surfaces treatments [11]. The "true value" of hardness is achieved by the "skill operator", using an "ideal standards hardness calibration machine" with the "ideal indenter" and applying the standards without substantial error [12]. Vickers hardness test method is performed by indenting the test material with a diamond indenter, in the form of a right pyramid with a square base and an angle $\alpha=136$ degrees $( \pm 0.5)$ between opposite faces, Figure 1, subjected to a load of 1 to $1000 \mathrm{~N}$. The full load is normally applied for 10 to $15 \mathrm{~s}$. The diagonal of the indentation left in the surface of the material, $d 1$ and $d 2$, are measured after removing the load and their average length is calculated. The area of the indentation surface is calculated. Vickers hardness value is obtained by dividing the applied load by the area of indentation using the following equation [11].

$$
\mathrm{HV}=\frac{2 F \sin \frac{136^{\circ}}{2}}{d^{2}}
$$




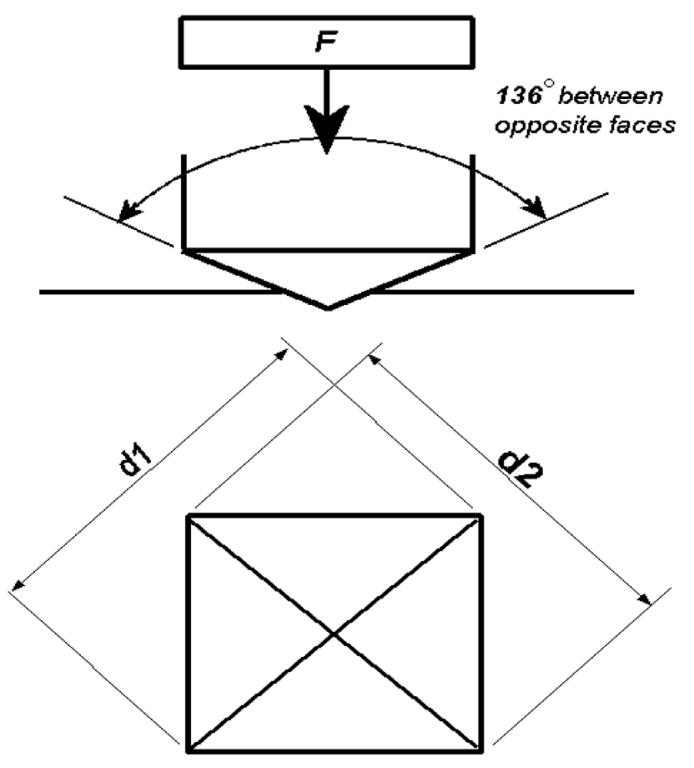

Fig. 1. Indentation geometry.

$\mathrm{HV}=$ Vickers hardness;

$F=$ load in kgf (force in Newton/gravity acceleration g);

$\alpha=$ the angle between the opposite faces of the pyramidal indenter $=136^{\circ} \pm 1$;

$d=(d 1+d 2) / 2 \mathrm{~mm}$.

In practice, it is impossible to realize the true value of the hardness scale, because the hardness machine and indenter cannot be manufactured and calibrated without tolerances. However, by controlling and reducing such tolerances, errors and their uncertainty contribution to the hardness scales can be minimized. It has been recognized that different indenters, of nominally similar geometry, can lead to large variations in measured hardness values [13]. Therefore, the work described in this paper aims to quantify the effect of different indenter geometries on measured Vickers hardness.

The error of the Vickers hardness due to the deviation in the angle between the opposite faces of the indenter is calculated from the partial differentiation [14] of equation (1) with respect to the angle $\alpha$ as follows:

$$
\frac{\partial \mathrm{HV}}{\partial \alpha}=\frac{F \cos \frac{\alpha}{2}}{d^{2}}
$$

where $F=490.3 \mathrm{~N}$ in this experiment.

\section{Experimentation}

The hardness tests were performed using an Instron universal hardness testing machine model testor/930 Figure 2, The machine load was calibrated against reference force transducer of uncertainty $\pm 0.02 \%$, While the optical magnification system was calibrated against Precision magnifier with mm scale calibrated against Carl Ziess Abbe-Comparator with uncertainty of $\pm 0.001 \mathrm{~mm}$. The angles of Vickers indenters $\alpha$ were measured in the two

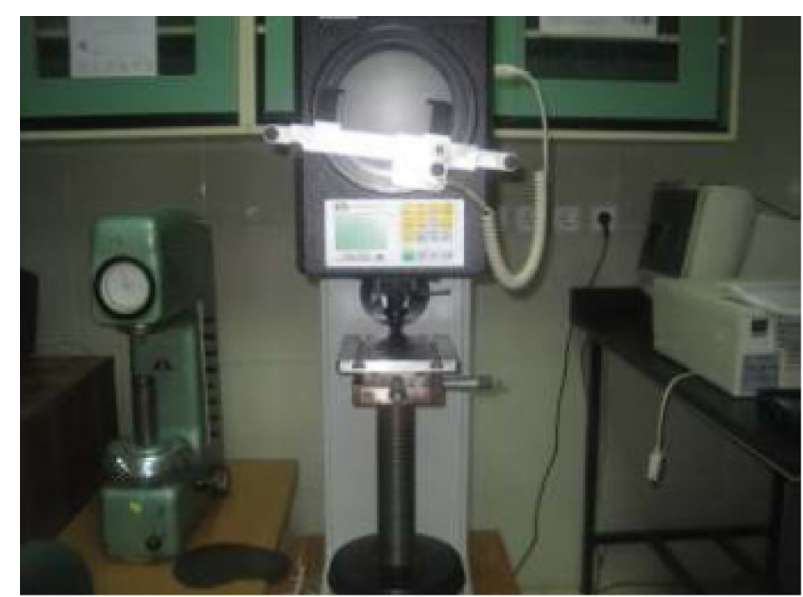

Fig. 2. (Color online) Instron hardness machine.

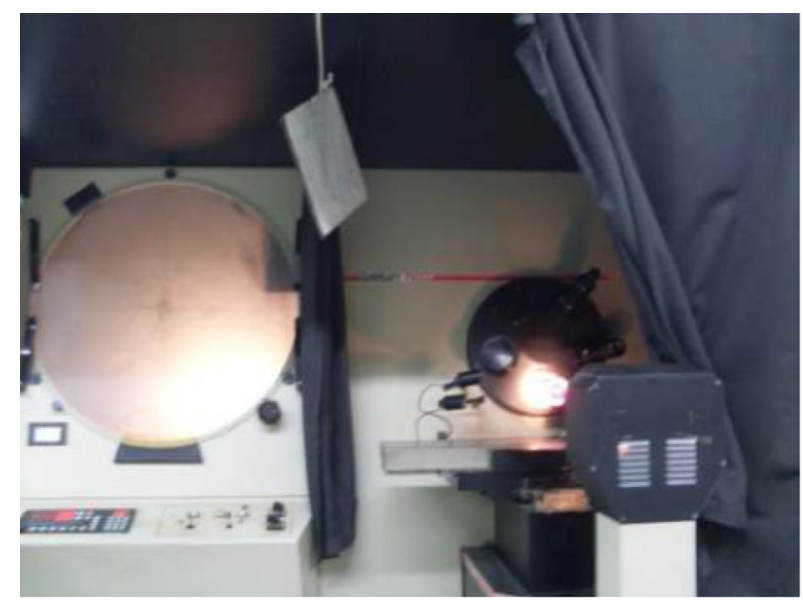

Fig. 3. (Color online) Optical comparator.

Table 1. Measured angles of the Vickers indenters.

\begin{tabular}{ccccc}
\hline $\begin{array}{c}\text { Indenter } \\
\text { serial } \\
\text { Number }\end{array}$ & \multicolumn{2}{c}{$\begin{array}{c}\text { The angles } \\
\text { between the two } \\
\text { pairs of faces } \\
\alpha 1\end{array}$} & $\begin{array}{c}\text { Average } \\
\text { values of } \\
\text { the angles } A\end{array}$ & $\begin{array}{c}\text { Line of } \\
\text { conjunction } \mu \mathrm{m}\end{array}$ \\
\hline 1 & 135.23 & 135.77 & 135.5 & \\
2 & 136.62 & 135.58 & 136.1 & $<1$ \\
3 & 136.23 & 136.44 & 136.335 & $<1$ \\
4 & 136.64 & 136.85 & 136.745 & $<1$ \\
5 & 136.12 & 136.47 & 136.295 & $<1$ \\
6 & 136.39 & 136.33 & 136.36 & $<1$ \\
7 & 136.89 & 136.72 & 136.805 & $<1$ \\
8 & 135.92 & 135.76 & 135.84 & $<1$ \\
9 & 136.45 & 136.89 & 136.67 & 4 \\
\hline
\end{tabular}

opposite directions by Quadra-check II optical comparator Figure 3 of uncertainty of $\pm 0.01^{\circ}$.

Nine indenters were selected. Angles between the pairs of opposite faces, $\alpha 1$ and $\alpha 2$ of each of the nine pyramidal indenters were measured. These are listed in Table 1 and the average values of the angles between the pairs of faces $\alpha$ are calculated. Also the line of conjunction of each indenter is presented. It can be seen that the indenter angles 
Table 2. Effect of indenter angles for HV measurement in 2 different reference HV blocks.

\begin{tabular}{ccc}
\hline Indenter angle $\left(^{\circ}\right)$ & \multicolumn{2}{c}{ Average Measured HV } \\
& Ref. HV 620 \pm 1 & Ref. HV $345 \pm 1$ \\
\hline 135.5 & 621.2 & 346.4 \\
136.1 & 620 & 345.4 \\
136.335 & 618 & 344.4 \\
136.745 & 616.2 & 342.2 \\
136.295 & 619 & 344 \\
136.36 & 617.2 & 343.4 \\
136.805 & 616.4 & 343.4 \\
135.84 & 621.4 & 345.8 \\
136.67 & 610.8 & 343.6 \\
\hline
\end{tabular}

vary from 135.5 to 136.805 . These indenters show a range of variation in $\alpha$ of $1.305^{\circ}$; which is $0.96 \%$ of the nominal angle. Most of the indenter angles and line of conjunction lies Each of the nine indenters of different face angles was used to perform hardness test on two different calibrated reference hardness blocks. The blocks have different hardness values of 620 HV50 and 345 HV50. The tests were performed according to the ISO/CD 5607-1 [15]. Each test was carried out with five indentations. The average of five indentations is obtained. within the tolerance specified by the ISO/CD 5607-1 [15].

Tests carried out under controlled conditions at a temperature of $(23 \pm 5){ }^{\circ} \mathrm{C}$.

The indenter contact with the test surface and apply the test force in a direction perpendicular to the surface, without shock or vibration, until the applied force attains $50 \mathrm{~kg}$. The time from the initial application of the force until the full test force is reached was $5 \mathrm{~s}$.

The approach speed of the indenter was $0.2 \mathrm{~mm} / \mathrm{s}$, and the indenter contact the test piece at a velocity between $40 \mu \mathrm{m} / \mathrm{s}$.

The duration of the test force was $15 \mathrm{~s}$.

The lengths of the two diagonals were measured. The arithmetical mean of the two readings was taken for the calculation of the Vickers hardness.

The results of measurement are listened in Table 2 while Figures 4 and 5 present the relationship between indenter angle and Vickers hardness values.

It is shown that the hardness value increases by the decreasing the indenter angle. Figure 4 shows that the angle variation of $1.308^{\circ}$ leads to corresponding Vickers hardness variation of $10.6 \mathrm{HV}$ i.e. about $1.7 \%$ for the hardness value of $620 \mathrm{HV}$. However, for the hardness value of $345 \mathrm{HV}$ in Figure 5, $1.308^{\circ}$ variation in indenter angle leads to corresponding Vickers hardness variation of 4.2 HV. It is shown from the experimental results that the standard error of estimate Se is about $2.1 \mathrm{HV}$ for $620 \mathrm{HV}$ hardness value, and $0.54 \mathrm{HV}$ for the $345 \mathrm{HV}$.

The best fit lines for the relationship are plotted with confidence level of $95 \%$. Dependence of HV values on the actual indenter angle size is readily seen. Figure 6 present deviation of the hardness value from the reference value due to indenter angle error. Equations (4) and (5) relate the Vickers hardness error due to angle error for $620 \mathrm{HV}$, and $345 \mathrm{HV}$ hardness value respectively, as obtained from

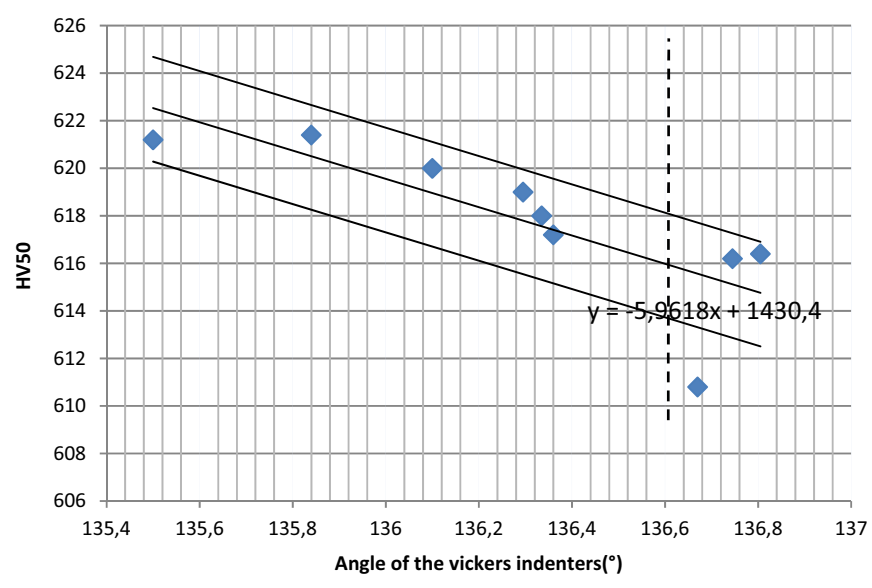

Fig. 4. (Color online) Relation between angle of the indenter and hardness value (620 HV50).

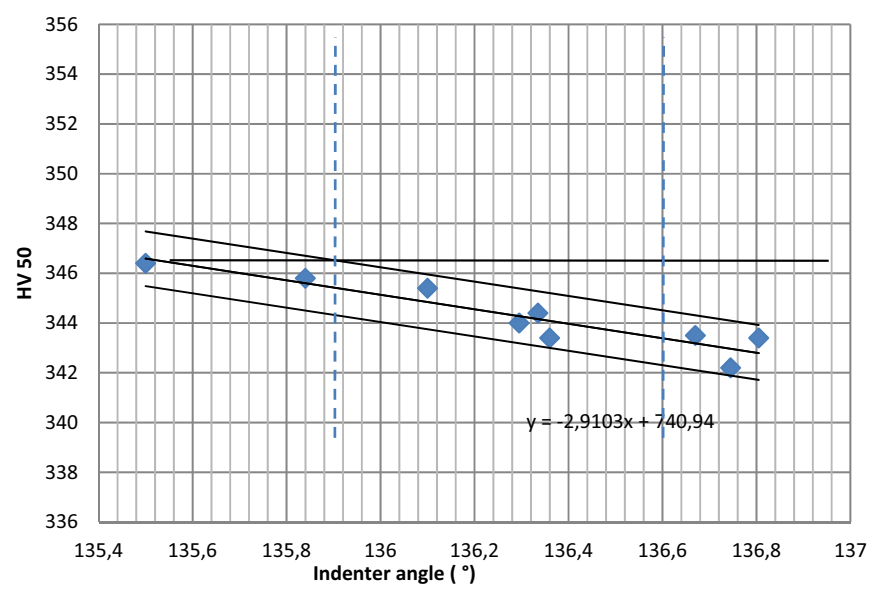

Fig. 5. (Color online) Relation between angle of the indenter and hardness No (345 HV50).

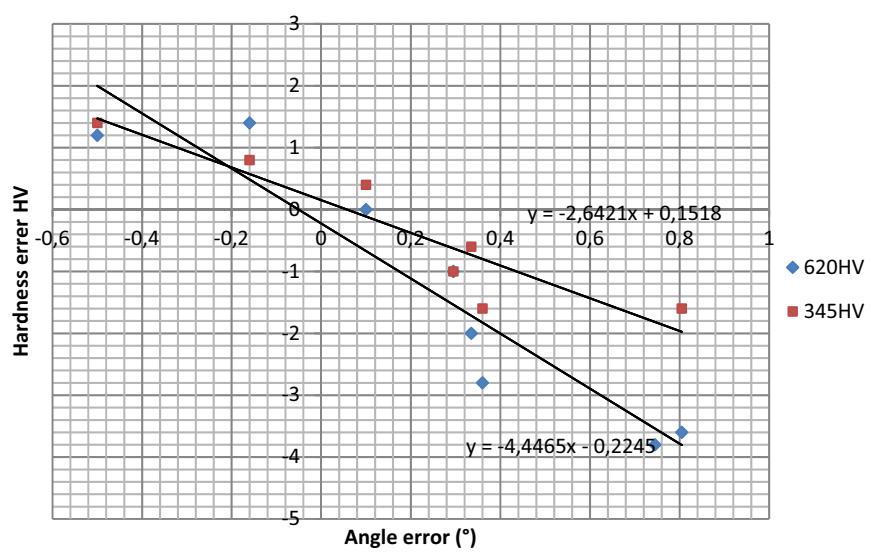

Fig. 6. (Color online) Hardness error due to angle error.

the best fit line given in Figure 6 .

$$
\begin{aligned}
& \mathrm{HV} \text { error }=-4.446 \times \text { angle error }-0.224 \\
& \mathrm{HV} \text { error }=-2.601 \times \text { angle error }-0.157 .
\end{aligned}
$$

The general equation for error $\%$ is obtained by dividing the two equations by the hardness number, and averaging 
the two constants

Hardness error $\%=-0.721 \times$ angle error -0.043 .

This means that every $1^{\circ}$ error in angle of the indenter gives a hardness error about $0.764 \%$.

Considering the indenter number 6677 with angle of $136.67^{\circ}$, it has the maximum line of conjunction $=4 \mu \mathrm{m}$. This particular indenter showed pronounced scattering in the results in the specimen of hardness value of $620 \mathrm{HV}$, because the small indentation size is more sensitive to geometrical error. This particular case revealed the importance of investigating the effect of geometrical tolerances on the length of the conjunction line and hence on the measured hardness values. This effect will be considered in separate investigation.

\section{Analytical approach}

For theoretical calculations, to study only the effect of indenter angle on the hardness number, the other factors which affect the hardness No (force and magnification) should assumed to be constants.

From the equation of the Vickers hardness if we differentiate the equation this lead to the following equation

$$
\begin{aligned}
\Delta \mathrm{HV}= & \frac{0.102 F \cos \frac{\alpha}{2}}{d^{2}} \Delta \alpha+\frac{0.204 \sin \frac{\alpha}{2}}{d^{2}} \Delta F \\
& -\frac{0.408 F \sin \frac{\alpha}{2}}{d^{3}} \Delta d .
\end{aligned}
$$

By assuming that is no error in force and magnification (constants) $\Delta F, \Delta d=0$ one can obtain,

$$
\Delta \mathrm{HV}=\frac{0.102 F \cos \frac{\alpha}{2}}{d^{2}} \Delta \alpha
$$

$d$ is $0.39 \mathrm{~mm}$ for the specimen with hardness value of $620 \mathrm{HV}$, and $d=0.54 \mathrm{~mm}$ for the specimen with hardness value of $345 \mathrm{HV}$. Hence from equation (7), every $1^{\circ}$ give corresponding error of $2.1 \mathrm{HV}$ for the hardness value of $620 \mathrm{HV}$, and 1.2 HV for the specimen with hardness value of $345 \mathrm{HV}$.

\section{Numerical modeling}

To investigate the effects of indenter shape on HV, finite element analyses [16] were carried out. The model used for these calculations is illustrated in Figure 7. Indentation into the center of the $10 \mathrm{~mm}$ length test specimen with a square cross section of $10 \times 10 \mathrm{mms}$ was considered (it should be noted that the size of the selected specimen will not affect the results). Assuming the indenter to be a rigid body, the sample was assumed to be an elastoplastic material. Material of the sample was chosen from metals of which properties are clearly known. The material used are steel 60 with yield strength of $400 \mathrm{Mpa}$ and tensile strength of $60 \mathrm{Mpa}$, and steel with yield strength of

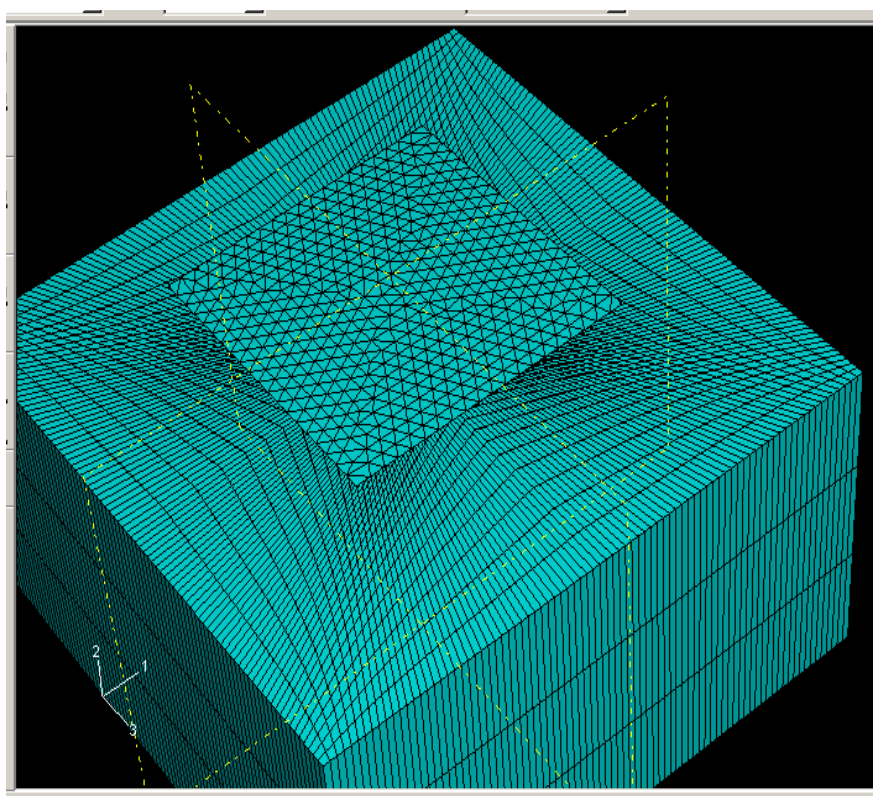

Fig. 7. (Color online) The finite element model for Vickers hardness test.

Table 3. Results of finite element analysis.

\begin{tabular}{ccc}
\hline Indenter angle $\left(^{\circ}\right)$ & Diagonal length $\mathrm{mm}$ & Hardness HV50 \\
\hline 135.5 & 0.3844 & 627.782 \\
136.1 & 0.3852 & 625.177 \\
136.336 & 0.3857 & 623.557 \\
136.745 & 0.3862 & 621.944 \\
136.295 & 0.3854 & 624.528 \\
136.36 & 0.3859 & 622.911 \\
136.805 & 0.3859 & 622.911 \\
135.84 & 0.3849 & 626.152 \\
136.67 & 0.3861 & 622.266 \\
\hline
\end{tabular}

$600 \mathrm{Mpa}$, and tensile strength of $850 \mathrm{Mpa}$ to give almost the same hardness values of two reference blocks 345 , and $620 \mathrm{HV}$. A model of pyramidal indenter was created by SolidWorks. Its shape illustrated in Figure 8. The computation was carried out by ABAQUS program.

The boundary conditions shown in Figure 9. The indenter is permitted to move in $z$ direction only, and the displacement $n x, y$ direction $=0$, while for the specimen, the displacement at the lower surface is zero for all directions $(x, y, z)$.

Using the same indenter angles used in the experimental works. The shape of the indentation obtained by the finite element analysis is illustrated in Figure 8, and the HV results obtained are presented in Table 3. Figure 10 shows the relationship between computed hardness errors due to angle error investigated by the finite element model.

From the finite element analysis as shown in Figure 9, the hardness error is related to the angle error by the equation:

$$
\text { Hardness error }=-4.319 \times \text { angle error }+0.398 .
$$

This means that every $1^{\circ}$ error in indenter angle leads to 4.717 HV. A noteworthy is that the finite element analysis 


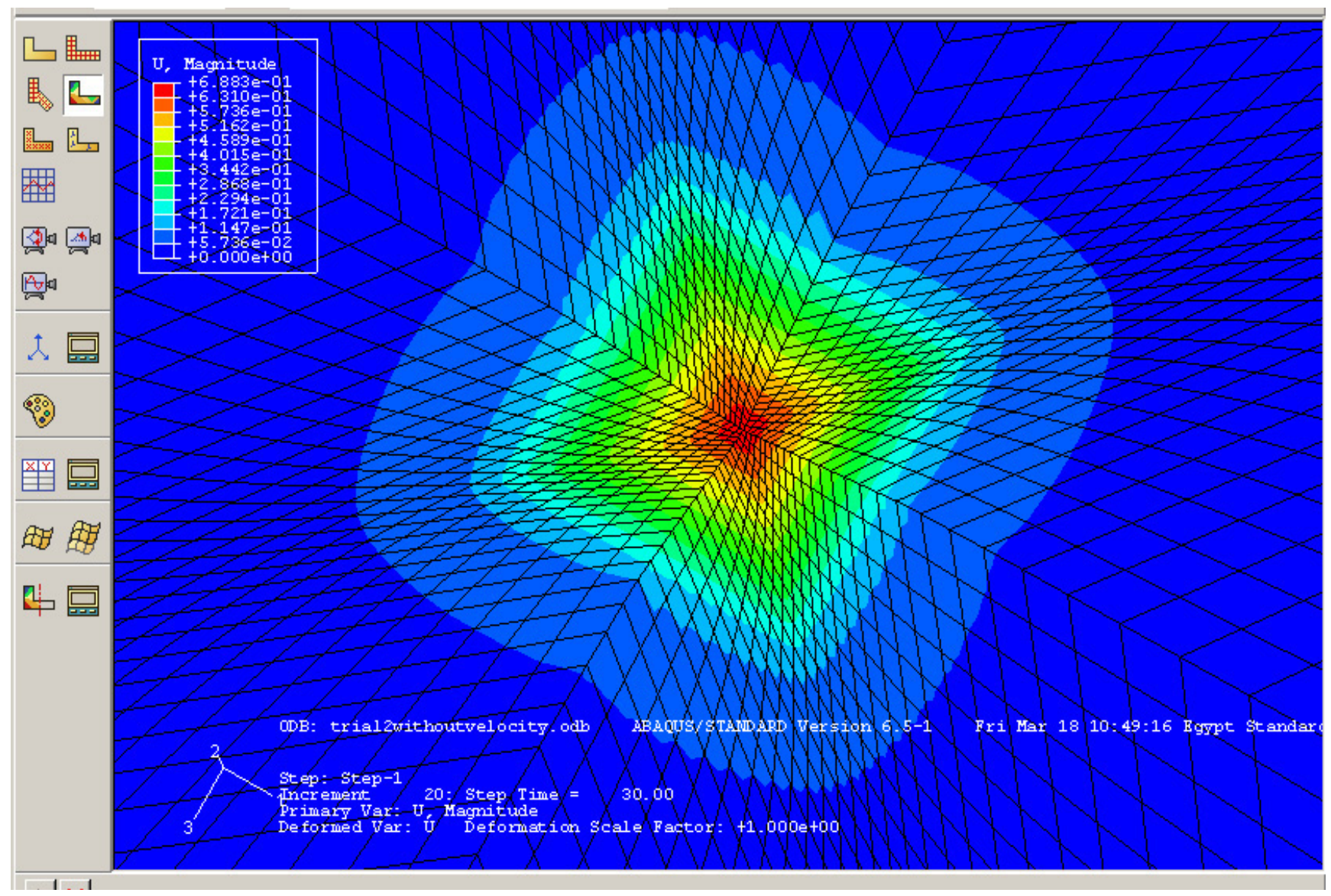

Fig. 8. (Color online) Indentation shape of the Vickers hardness.

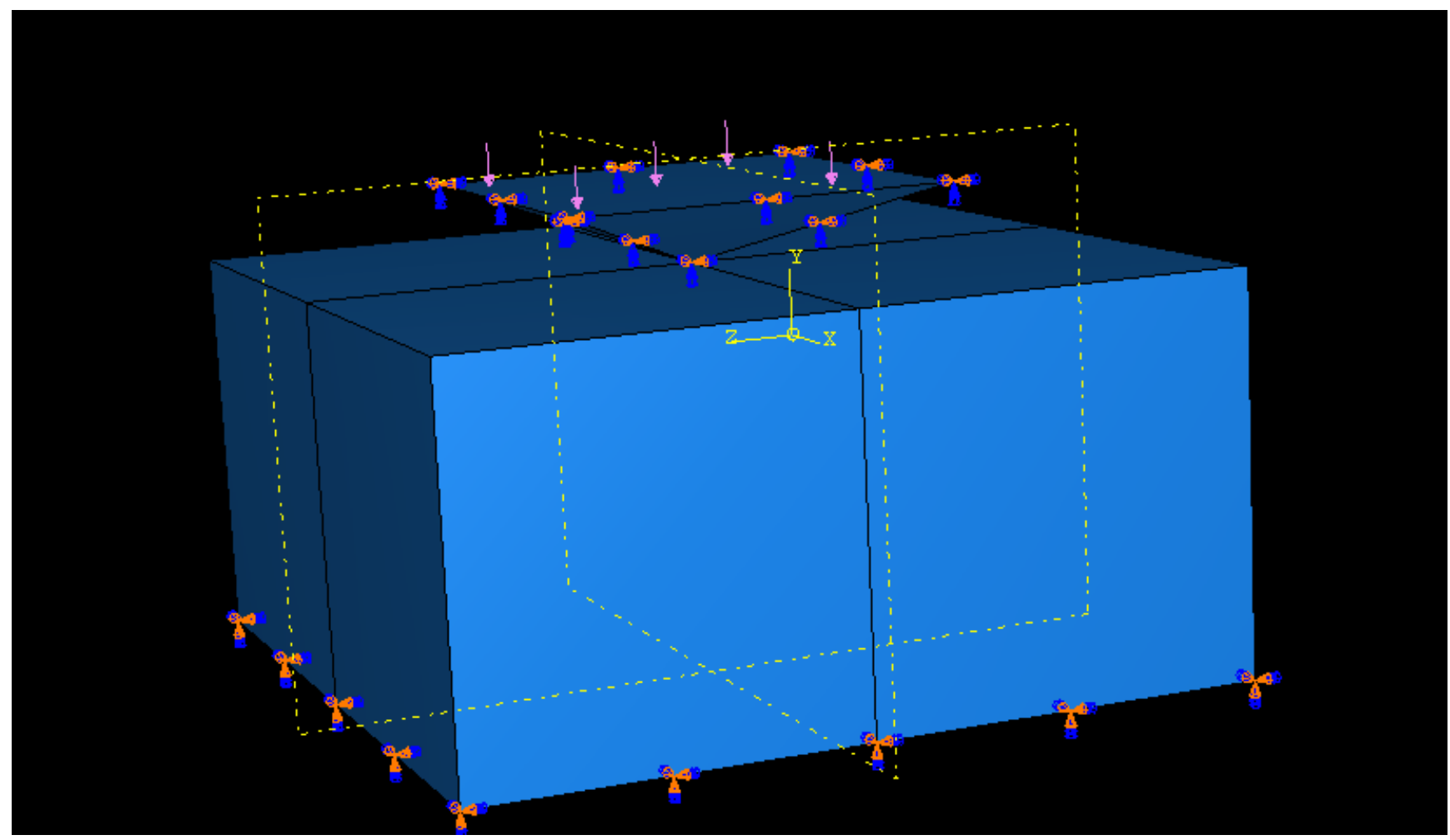

Fig. 9. (Color online) Boundary condition for Vickers hardness. 


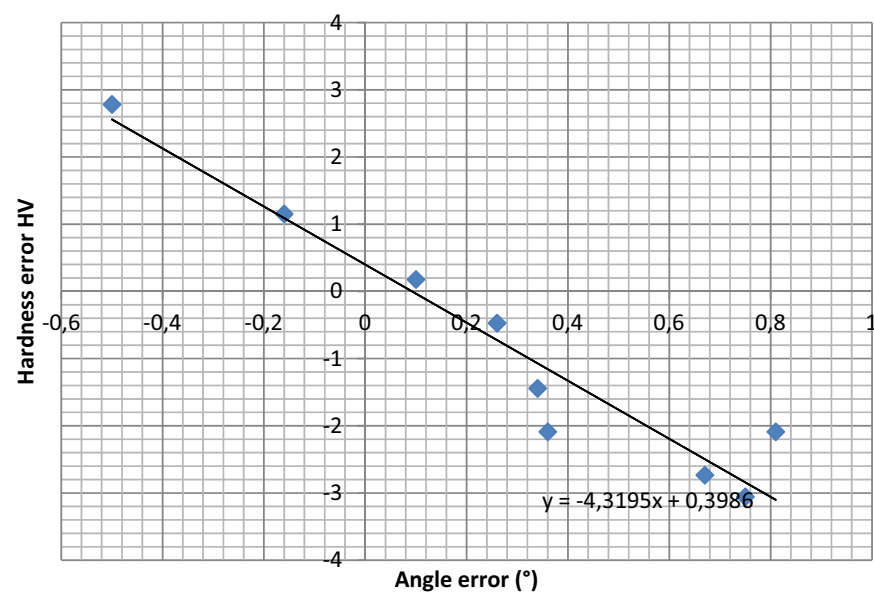

Fig. 10. (Color online) Hardness errors due to angle error.

Table 4. Comparison between experimental, theoretical, and numerical analysis.

\begin{tabular}{ccc}
\hline $\begin{array}{c}\text { Experimental } \\
\mathrm{HV} /\left(^{\circ}\right)\end{array}$ & $\begin{array}{c}\text { Theoretical } \\
\mathrm{HV} /\left(^{\circ}\right)\end{array}$ & $\begin{array}{c}\text { Finite element } \\
\mathrm{HV} /\left(^{\circ}\right)\end{array}$ \\
\hline 4.67 & 2.1 & 4.717 \\
\hline
\end{tabular}

yields both elastic and plastic deformations under load. The diagonal $\mathrm{d}$ is calculated from $x$ and $y$ coordinates of the corner points after taking the load off, which implies that the diagonal is calculated for the permanent plastic deformation after the spring back of the elastic component of the displacements.

Table 4 presents comparison between experimental, theoretical, and numerical analysis for the error of $\mathrm{HV}$ due to error on indenter angle. It is seen that the finite element analyses agrees well with the experimental results which is almost duple the theoretical results, because of other sources of error which is not considered in the theoretical calculations, such as force, magnification, and the asymmetry of the angle between the two opposite faces, which is considered in the finite element analysis. Furthermore, differences between the experimental and numerical results can be attributed to the effect of the surface roughness of the indenter faces which was not considered in the numerical solution.

\section{Conclusion}

The present paper considered the effect of the indenter angle on the Vickers hardness value. A few remarks can be concluded:

1- In Vickers hardness, the measured hardness value was overestimated with decreasing the indenter angle. This implies that negative tolerances on the indenter angle results in apparent increased hardness, while positive tolerances and errors will result in apparent lower hardness values.

2- An equation which govern the relation between the angle error and hardness error can be extracted from the present results:

Hardness error $\%=-0.721 \times$ angle error -0.043 , which indicates that every $1^{\circ}$ error in angle of the indenter gives a hardness error of $0.764 \%$.

3- The variations in indenter angle of the Vickers hardness, while within the limits allowed by the ISO standard, can lead to variations in measured hardness, which may exceed permissible values. Therefore, it is recommended that the tolerance in Vickers indenter angle in the ISO standard be more tightened to less than $\pm 0.5^{\circ}$.

\section{References}

1. J.G. Wood, J. Cotter, P.J. Nash, Hardness testing - A survey of Rockwell C scale diamond indenters, NPL Report MOM 40, 1980

2. G. Barbato, S. Desogus, A. Germak, Report on the results obtained in the comparison of the HRC scales maintained at ETCA, IMGC and MPA NRW, Rapporto Tecnico Interno R 382, IMGC, Torino, 1994

3. R.S. Marriner, J.G. Wood, Investigation into the measurement and performance of Rockwell $\mathrm{C}$ diamond indenters, Metallurgia $8 \mathbf{7}$ (1967)

4. G. Barbato, S. Desogus, A. Germak, The development of hardness scales in Europe, Measurement Science conference (Anaheim, CA, 1992)

5. P. Key, P. Soardo, The European cooperation on calibration testing and certification, Proc. of cooperation in metrology equivalence of the national standards dissemination of SI units, Torino, Italy, 1992, pp. 62-85

6. J.G. Wood, J. Cotter, Performance predictions of Rockwell indenters, NPL Report MOM 73, 2000

7. J.G. Wood, J. Cotter, Performance predictions of Rockwell indenters, NPL Report MOM 73, 1999

8. J.F. Song, S. Low, D. Pitchure, A. Germak, S. DeSogus, T. Polzin, H.Q. Yang, H. Ishida, Establishing a common Rockwell hardness scale using geometrically calibrated standard diamond indenters, Proc. XIV IMEKO World Congress, Tampere, Finland, 1999

9. J.F. Song, S. Low, D. Pitchure, A. Germak, S. DeSogus, T. Polzin, H.Q. Yang, H. Ishida, G. Barbato, Establishing a world-wide unified Rockwell hardness scale with metrological traceability, Metrologia 34, 331-342 (2002)

10. J.F. Song, T.V. Vorburger, Standard grade Rockwell diamond indenters - A key to a worldwide unified Rockwell hardness scale, Proc. of National Conference of Standard Laboratories (NCSL, CA, 1996), pp. 403-417

11. H. Czichos, Springer Handbook of materials measurements methods (Springer, 2006)

12. L. Brice, Uncertainty in hardness measurements, NPL Report CMAM 87, 2003

13. L. Brice, The influence of indenter characteristics on hardness measurements, XVII IMEKO World Congress Metrology in the 3rd Millennium, Dubrovnik, Croatia, 2003

14. J. Petrík, The influence of the ball on the Brinell hardness Tester calibration, Mapan - Journal of Metrology Society of India 23, 197-205 (2008)

15. ISO/CD 5607-1, Metallic materials-Vickers hardness testtest method, 2005

16. O.C. ZienKiewicz, The finite element method, 5th edn., Solid mechanics (1999), Vol. 2 